\title{
The Role of Team: Analysis on Hotspots, Dimensions and Model of Knowledge-based Team Human Capital
}

\author{
Zenghai Wu ${ }^{1, a}$, Beijie Yin ${ }^{1, ~ b, ~}{ }^{*}$, Jin Yang ${ }^{1, c}$ \\ ${ }^{1}$ Shaanxi Normal University, Xi'an, Shaanxi, China \\ awuzenghai@snnu.edu.cn, bbelleybj@163.com, c2236629111@qq.com \\ * Corresponding author
}

Keywords: Human Capital, Team, Bibliometric Analysis, Review

\begin{abstract}
In the context of organizational flattening and teamwork, human capital at the team level will be more achievable than individual human capital incentives, so the organization is more inclined to the value-added of human capital development on the overall level of the team. The research and development of the team human capital are also urgent. Firstly, based on the CitespaceV software for the past ten years of domestic and international bibliometric and hotspots analysis, this paper summarizes dimension division of team human capital of knowledge-based teams, and forms its element structure including 2 dimensions and 5 elements. Secondly, this paper forms a basic framework model of team human capital: The antecedent variables include individual factors and interaction factors, the adjustment variables are team organizational factors, and the outcome variables are divided into four factors: overall and innovation performance, related inputs and decision-making. Finally, according to the above summary, it is pointed out that the future research should focus more on the formation path and dynamic changes of team human capital.
\end{abstract}

\section{团队的作用：知识型团队人力资本的研究热点、维度与模型}

\author{
武增海 1 , a, 尹蓓婕 $1, \mathrm{~b},{ }^{*}$, 杨瑾 $1, \mathrm{c}$ \\ 1 陕西师范大学国际商学院, 西安, 陕西, 中国 \\ awuzenghai@snnu.edu.cn, bbelleybj@163.com, c2236629111@qq.com \\ “通讯作者
}

关键词: 人力资本; 团队; 文献计量分析；综述

中文摘要. 在组织扁平化和团队合作的大背景下, 相比于个体的人力资本激发, 团队层面的人 力资本将更加可以直接为知识型组织所用，因此组织更倾向于团队整体层面上的人力资本开 发增值, 有关团队人力资本的研究和发展也已迫在眉睫。本文在使用 CitespaceV 软件对知识 型团队人力资本进行近十年来国内外文献计量和热点分析的基础上, 对其现有维度划分方式 进行了归纳梳理, 得出 2 维度、5 要素的团队人力资本要素构成结构, 从而形成团队人力资 本基本框架模型：前因变量包括个体因素和交互因素, 调节变量为团队组织因素, 结果变量 为总体与创新绩效、相关投入和决策等四因素。最后根据上文的总结梳理，指出未来研究重 点在于对团队人力资本形成路径与动态变化的研究。

\section{1.引言}

Ployhart 等提出, 组织内部存在分层次的剖面特征, 个体、团队、组织作为组织内部的不 同层次，相互嵌套、相互影响 ${ }^{11]}$ 。为了使个体层面人力资本汇集到团队层面，就需要发挥团 
队 $1+1>2$ 的效果, 对于团队人力资本提升的研究也就刻不容缓。在实际的管理实践中，才队 人力资本的存在是冊庸置疑的。

首先, 在当今技术快速发展和市场竞争激烈的背景下, 个体独立地完成工作愈加困难 ${ }^{[2] 。}$ 而越来越多团队的出现减轻了个体工作的风险性，提高了整体工作的准确性和全面性，其原 因正是作为团队层面的人力资本在发挥着作用。其次，越来越多的企业出现了“集体跳槽”的 现象, 而且往往是企业内的中高层集体离职, 对企业的发展打击极大。这种现象往往存在于 项目型企业中，研发团队、高管团队、销售团队等都具有集体性，一个人跳槽将带走团队的 其他人，比较著名的就是英特尔研发团队集体跳槽至中科院，这正说明了团队人力资本确实 存在且具有完整性和一定的排他性。第三，企业的薪酬激励制度从个人激励逐渐转为团队激 励, 也可以看出团队人力资本的存在。针对团队整体的产出和绩效进行奖励, 所得到的激励 效果比个人激励更大, 这也正说明了团队人力资本的存在及其重要性 ${ }^{[3]}$ 。最后, 企业内部结 构扁平化、越来越多的团队代替部门制的现状也促使管理者们重视团队层面上的人力资本持 有、增值的问题。企业中越来越多的团队取代着独立的个体，对团队人力资本的重视将实现 团队绩效的提高，进而实现组织绩效的提高。

团队人力资本的存在，意味着组织应当摆脱个体的限制，不仅仅局限于某一个或某几个 个体, 而更应该着眼于团队层面, 从团队的高度提升整体人力资本, 从而实现 $1+1>2$, 如此 才能让团队真正发挥价值。另外, 由于知识型团队的人力资本附加值较高、更具有参考价值, 因此一般意义上讲到的团队人力资本载体就是知识型团队 (包括研发、高管、项目等团队), 本文也将对其进行重点关注。

\section{2.基于文献计量的团队人力资本研究热点分析}

在中文文献分析中，笔者利用中国知网（CNKI）数据，以关键词 or 主题词=“团队人力 资本”进行检索, 在去除书评、征稿通知等不符合要求的文章后, 将原始数据进行去重和格式 转换, 最终得到近十年 (2009 年至今) 文章 179 篇; 在外文文献分析中, 利用 web of science 数据, 以关键词 or 主题词= “team human capital”进行检索, 经过上述的同样篮选后, 得到相 关文章 770 篇。

CitespaceV 是一款基于 Java 环境、对文献进行可视化分析的文献处理工具。本文使用 CitespaceV 5.3.R4, 对上述检索到的文献进行热点分析, 中文文献分析的结果如下图 1 所示, 外文文献分析的结果如下图 2 所示。在 CitespaceV 的共现图中, 节点越大, 说明该关键词在 所选文献中出现的次数越多; 词与词之间的连线说明关键词间的两两关系。通过软件的计量 后得到的关键词共现图, 可以有效地反映这一领域中出现频次最高的词汇与问题, 进而反映 出整个领域的关注热点。

有关团队人力资本近十年的中外研究热点表明, 团队性质上, 研究主要集中于高管团队、 研发团队和创业团队, 其中高管团队是最为热点的研究对象, 而农村人力资本团队则是最新 的研究热点所在; 在团队人力资本的影响研究中, 目前研究大多仍与团队绩效和企业总体或 创新绩效相结合，并在此过程中直接或间接影响着企业的特定投入（如研发投入）并最终影 响企业在市场中的竞争优势; 而在横向比较中, 团队人力资本作为人力资本的一部分, 往往 与社会资本和心理资本相互影响、共同作用。通过上述文献的计量结果，可以更加明确未来 团队人力资本的发展方向，并为下文的有关变量列出提供借鉴。 


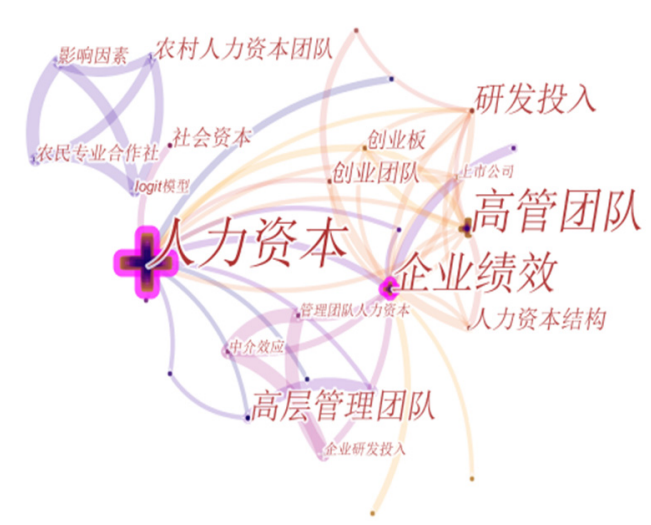

图 1 基于 CNKI 数据的关键词共现图（关键词 or 主题词=“团队人力资本”）

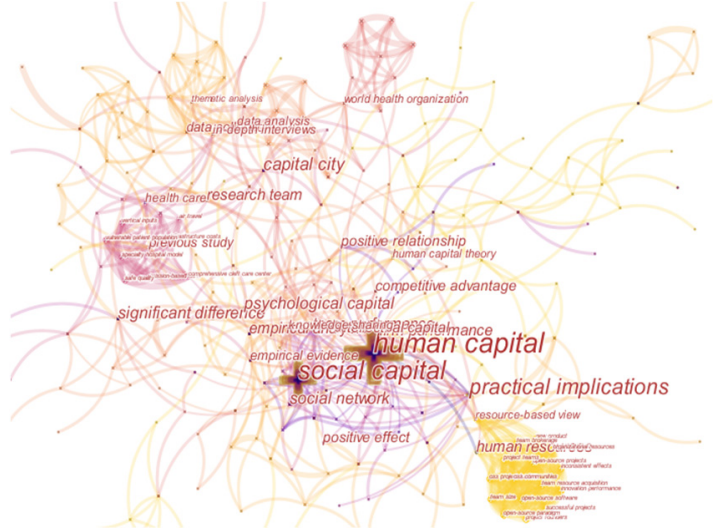

图 2 基于 WOS 数据的关键词共现图（关键词 or 主题词=“team human capital”)

\section{3.团队人力资本维度划分及测量}

\section{1 团队人力资本维度划分述评}

葛玉辉将团队人力资本分为五个维度进行测量：（1）贡献，即业绩、经验、员工培养等; （2）合作，包括团队合作和凝聚力;（3）努力, 即为生产和合作所做出的努力; (4) 能力, 包括团队中需要的人际沟通、学习、协调、分析、控制、领导等能力;（5）基本素质，包括 个人健康、知识储备、智商、职业技能等 ${ }^{[4]}$ 。鲁虹也据此, 将其分为个体和团队两个层面, 个体人力资本包括心智模式、才能、努力程度, 团队层面就包括协作能力、集体创新能力、 共担风险以及继续成长的能力 ${ }^{[5]}$ 。总体而言, 这种分类方式构建了一个团队人力资本维度结 构的大框架, 理清了团队人力资本内部的区分情况, 但具体的测量如努力程度等难以量化, 在测量上存在不足之处。

Laszlo 等、Richard 等和 Khanna 等都以人口统计特征为出发点, 将学历、职称、年龄等 的百分比作为团队人力资本的测量指标 ${ }^{[6-8]}$, 而朱炏等则在此基础上将团队人力资本划分为传 记性和非传记性两种, 前者即年龄、任期等的均值, 而后者则指建立在传记性之上的年龄、 任期等的异质性 ${ }^{[9]}$ 。这两种类型的人力资本可以分别使用均值和标准差的方式直接进行测量, 进一步扩充了团队人力资本的研究, 但存在的问题是仅注意到了比较浅层的、以人口统计变 量为基础的人力资本类型，而忽视了上文提到的成员能力、团队协作力等内容，比较片面。

鲁虹等再次对团队人力资本进行维度划分，以高管团队为依托，将团队人力资本分为能 力性人力资本和意愿性人力资本两种：前者指存在于高管团队中的知识、技能、社会关系网 络、声誉等方面的价值存量, 包括年龄、受教育程度、社会关系等; 后者则顾名思义, 回答 的是团队是否愿意为实现企业目标而贡献价值的问题，包括组织忠诚度、内驱力等 ${ }^{[10]}$ 。这种 分类方法除顾及比较显性、可观测的人力资本外, 还较为科学地考虑到成员与团队的主动性 
意愿，且均有成熟量表得以进行有效度量，因此是一种比较合理的分类方式。

胡望斌等则从创业团队角度出发, 将团队人力资本的异质性分为社会性异质性和功能性 异质性：社会性异质性多与团队和企业本身工作无关，有较强的外部性，指学历、年龄、受 教育程度等; 而功能性异质性就有较强的内部性, 包括与工作相关的经验、偏好等 ${ }^{[11]}$ 。张莉 莉等将这种分类方法延伸到更广泛的团队中，认为团队人力资本应分为显性和隐性两种，显 性人力资本即时可直接进行测量的成员年龄、性别、学历百分比，对应社会性; 隐性则指对 价值创造有直接影响的能力、素质、技能等指标，对应功能性 ${ }^{[12]}$ 。这种分类方式是依照是否 与工作直接相关进行的分类, 有一定的科学性, 目前大多数的团队人力资本研究也多采取这 一种分类方式，将其分为显性与隐性两种分别度量，可操作性较强。

综合以上几种划分方式可以看到, 团队人力资本的维度划分主要分为四个方向: 一个方 向是按照个体、团队、组织的层次不同进行的划分，由小及大、从微观到宏观，这样的分类 的逻辑性较强, 且在相关变量和问题处理上, 由于存在层次性的特点, 因而将更加易于进行 分析; 但是其不足在于, 每个层次上的人力资本具体细分不明, 相对而言比较笼统。第二个 方向则是将团队人力资本分为传记性和非传记性两种：前者指团队所有成员年龄、学历、工 作经验等的均值, 用平均数进行测量; 后者则指团队成员的年龄、学历等的异质性，用标准 差来进行测量。这种分类方式将团队人力资本进行了充分的量化，但仅关注于表面浅层的人 口统计变量因素, 而忽视了团队协作、信任等更深层次的团队人力资本要素。第三个方向则 是按照人力资本各要素的不同性质进行划分，即分为能力型、意愿型、人口统计型等，这种 划分方式相对比较细致，但是缺乏一个合理有效的度量工具进行有效的测度，且比较有针对 性，如能力型人力资本中创业团队需要测量“闯”的开拓精神，而处于成熟期的企业团队则更 需要关注创新、协调能力以及“守”的能力, 这也就仅对于某一种或某几种团队有意义, 因此 该分类方式在推广上可能存在一定的难度。第四个方向是根据工作相关程度进行的划分，与 工作直接相关的经验、能力等被视为隐性，与工作无直接相关关系但对团队绩效有影响的因 素则被认为是显性的人力资本。例如，针对于工程项目团队的团队人力资本而言，其显性人 力资本就是团队成员的年龄、学历以及经验 (用在工程建筑行业的工作年限表示)，隐性人力 资本就是成员的团队协作能力、团队凝聚力等。由于该分类方法弥补了上述三种分类方式的 不足，分类较全面的同时具有可操作性，因此这种分类方法目前已被广为接受和认可。

\section{2 团队人力资本要素测量}

本文结合前人的研究成果, 认为知识型团队人力资本的维度可以分为显性和隐性两方面: （1）显性团队人力资本包括团队成员的年龄、学历、经验 (企业工作年限和行业工作年限), 反映团队基本的胜任素质;（2）隐性团队人力资本主要指团队凝聚力这一不易直接进行测量 的因素，间接影响成员的贡献意愿，反映团队作为一个整体的效能状况。

在针对上述两种类型的团队人力资本测量时, 笔者认为, 可以分为两种方法进行测量: 第一种方法用于直接测量年龄、学历等显性团队人力资本, 这些传记性特征往往需要考虑它 们的异质性，因此将这些特征作为定序变量进行测量，并使用标准差的方法实现异质性的度 量, 即标准差越大, 异质性越大。第二种方法用于测量隐性团队人力资本, 因该要素属于潜 变量, 无法直接进行测量, 因此需要利用量表对其进行测度：团队凝聚力的测量一般使用 $\mathrm{K}$. B. Henry 等提出的三维度 15 题项量表 ${ }^{[13]}$ 。在上述测量完成后, 除了使用常用的数据处理软件 进行分析外, 考虑到团队和个体层面测量的跨层次作用, 还需要使用 HLM 软件以进行个体 聚合到团队的多层次回归分析。

\section{4.团队人力资本模型构建}

结合上述分析，构建团队人力资本的基本框架模型，如下图 3 所示。前因变量包括个体 和交互两方面, 调节变量主要为团队层面因素, 最后结合上文关键词共现图, 给出包括总体 
绩效、创新绩效、相关投入、决策等四方面结果变量。个体人力资本、心理契约影响团队成 员对团队做出贡献的能力强度, 成员间的信任程度会影响团队人力资本中凝聚力的形成, 而 知识共享意愿则会导致成员贡献程度的不同 [14-16]。调节变量中，团队生命周期、绩效考核方 式和团队氛围的好坏，也会在团队人力资本的形成路径中起到双向的调节作用 ${ }^{[17-18]}$ 。最终， 团队人力资本的存量和增速，将决定或影响团队的总体和创新绩效、组织相关投入与决策 [19-20]。

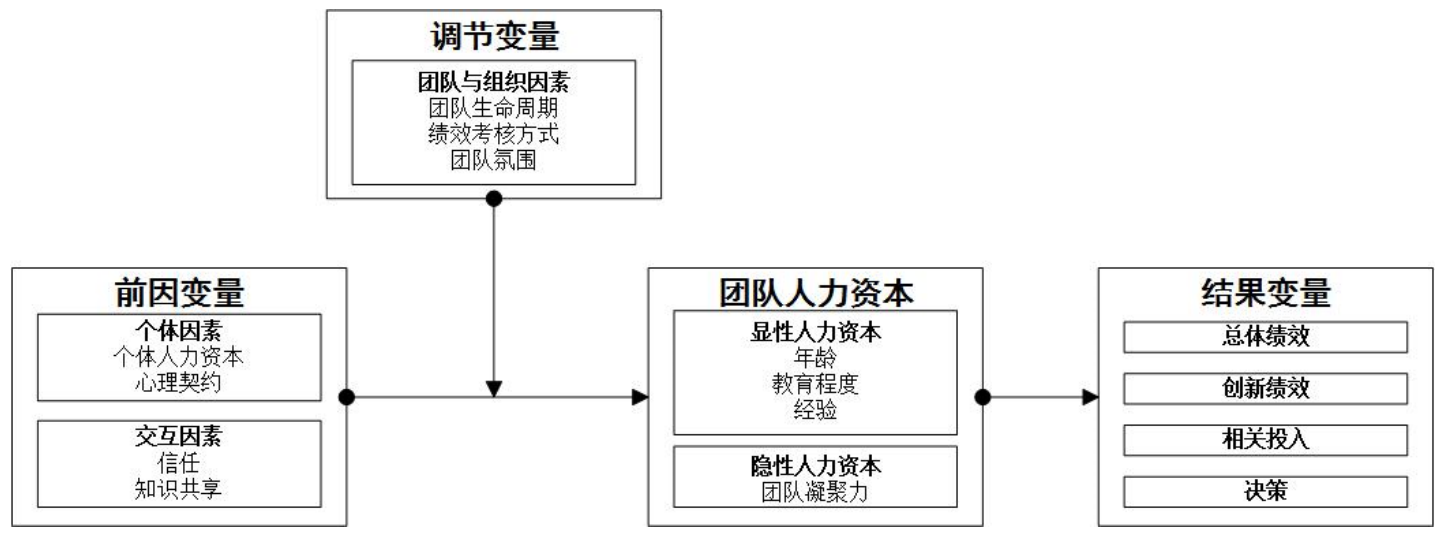

图 3 团队人力资本模型

\section{5.结语}

本文的贡献和创新点在于:（1）利用文献计量分析软件，分析团队人力资本的研究热点 所在, 而这一工作是前人未涉猎过的;（2）对团队人力资本维度研究现状进行分析后, 归纳 出四类维度划分方法, 有助于后人的继续研究; (3) 在梳理前人研究内容和进展的基础上提 出团队人力资本的框架模型, 这一模型可以为团队人力资本的下一步提供理论框架性的指导。

经过上述研究分析，笔者认为，未来团队人力资本的研究方向有两点可以进一步拓宽： (1) 注重对其形成过程和其前因变量的研究, 从而更好地把握团队人力资本的形成路径, 为 团队与组织发展提供理论参考; (2) 团队人力资本的动态变化问题值得深入探讨。事实上, 新成员加入和老成员退出都会对团队人力资本形成与增值产生双向的影响, 因此未来需要在 动态调整的环境下，进行更深入的团队人力资本增值方法路径的研究。

\section{致谢}

本文为陕西省社科基金项目《产学研促进陕西高新区协同技术创新问题与对策研究》 (2014D12) 和 陕西省软科学研究计划项目《大数据环境下科技创新团队人力资本激励与政 策设计》（2015KRM096）的阶段性成果之一。

\section{References}

[1] R. E. Ployhart and T. P. Moliterno, Emergence of the human capital resources: A multilevel model, Academy of Management Review, vol. 36, pp. 127-150, 2011.

[2] Z. L. Shi, Collaborative work and team building, China Soft Science, vol. 18, pp. 80-83, 2003.

[3] G. Duan, Z. Yang and B. Xu, Team-based reward, two-dimension sharing behaviors and knowledge team effectiveness, Science of Science and Management of $S$. \& $T$, vol. 36, pp. 160-170, 2015.

[4] Y. H. Ge, Research of the relationship model between top management team and enterprise performance based on human capital value factors, Science of Science and Management of S. \& T, vol.28, pp. 160-165, 2007. 
[5] H. Lu, Y. H. Ge, Top management team human capital and its evaluation model, Commercial Research, vol. 52, pp. 84-87, 2009.

[6] T. Laszlo, E. E. Alan, M. D. Catherine and R. D. Dan, Composition of the top management team and firm international diversification. Journal of Management, vol. 26, pp. 1157-1177, 2000 .

[7] C. R. Orlando and M. S. Roger. Linking top management team age heterogeneity to firm performance: juxtaposing two mid-range theories. International Journal of Human Resource Management, vol. 13, pp. 958-974, 2002.

[8] T. Khanna, J. Song and K. Lee, The paradox of Samsung's rise, Harvard business review, vol. 89, pp. 142-147, 2011.

[9] Y. Zhu and M. C. Zhang, Enterprise management team human capital, R\&D investment and enterprise performance, Accounting Research, vol. 34, pp. 45-52, 2013.

[10]H. Lu, X. Q. Li and Y. N. Xing, Research on top management team human capital on enterprise growth-based on empirical study listed companies on GEM, Science and Technology Management Research, vol. 34, pp. 157-162, 2014.

[11]W. B. Hu, Y. L. Zhang and J. Yang, Is homogeneity or heterogeneity? : A study on the adjustment function of the relationship between the technical entrepreneurial team and new ventures' performance, Management World, vol. 30, pp. 92-109, 2014.

[12]L. L. Zhang and X. P. Hu, "Team-working object" matching model based on human capital competition for optimal first structure, Journal of Industrial Engineering and Engineering Management, vol. 29, pp. 1-7, 2015.

[13]K. B. Henry, H. Arrow and B. Carini, A tripartite model of group identification: Theory and measurement, Small Group Research, vol. 30, pp. 558-581, 1999.

[14]L. Q. Wei and Z. H. Wang, Empirical study on the relationship between characteristics of TMTs and firm performance of Chinese businesses, Nankai Business Review, vol. 11, pp. 16-22, 2002.

[15]X. H. Zhu, C. Q. Yang, Q. H. Tan and J. W. Zou, Impact of psychological contract breach on individual creativity in university research team, Science and Technology Management Research, vol. 37, pp. 172-181, 2017.

[16]J. N. Guo, S. Y. Hao and Y. Li, Research on dynamic Evolution of knowledge transfer within construction project team, Journal of Engineering Management, vol. 32, pp. 121-125, 2018.

[17]M. L. Hou and H. Sun, The relationship between human capital and firm performance based on life cycle perspective, Enterprise Economy, vol. 37, pp. 104-111, 2016.

[18]M. Hernanez, D. R. Avery and S. Tonidandel, The role of proximal social contexts: assessing stigma-by-association effects on leader appraisals, The Journal of Applied Psychology, vol.101, pp. $68-85,2016$.

[19]C. Y. Gao and M. Wu, Study on the effects of top management team heterogeneity on firm performance: The moderating role of pay gap, Science-Technology and Management, vol. 21, pp. 1-8, 2019.

[20]X. R. Wang and W. Chi, Relationship between human capital heterogeneity of top management team and firm's innovation input: Moderating effect of status inequality among executives, Technology Economics, vol. 37, pp. 35-42, 2018. 